\title{
Crossover from a three-dimensional to purely two-dimensional vortex-glass transition in deoxygenated $\mathrm{YBa}_{2} \mathrm{Cu}_{3} \mathrm{O}_{7-\delta}$ thin films
}

\author{
Z. Sefrioui and D. Arias* \\ Departamento de Física Aplicada III, Universidad Complutense de Madrid, 28040 Madrid, Spain \\ M. Varela \\ Departmento de Física, Universidad Carlos III de Madrid, Leganés 28911 Madrid, Spain \\ J. E. Villegas \\ Departamento de Física Aplicada III, Universidad Complutense de Madrid, 28040 Madrid, Spain \\ M. A. López de la Torre \\ Departmento de Física Aplicada, Universidad de Castilla-La Mancha, 13071 Cuidad Real, Spain \\ C. León, G. D. Loos, and J. Santamaría \\ Departamento de Física Aplicada III, Universidad Complutense de Madrid, 28040 Madrid, Spain
}

(Received 20 May 1999; revised manuscript received 16 July 1999)

\begin{abstract}
Current-voltage $(I-V)$ characteristics were used to investigate the response of the vortex-glass (VG) phase transition in high-quality epitaxial $\mathrm{YBa}_{2} \mathrm{Cu}_{3} \mathrm{O}_{7-\delta}$ films in magnetic fields up to $7 \mathrm{~T}$. We show that varying the oxygen content, the scaling analysis reveals a crossover from three-dimensional (3D) to a pure $2 \mathrm{D} V \mathrm{VG}$ transition with $T_{g}=0$. At small oxygen deficiencies $(7-\delta=6.75)$, the $\rho$ - $j$ curves scale according to the 3D VG model, which cannot be distinguished from a Bose-glass model from a scaling analysis with the magnetic field applied parallel to the $c$ axis. At a lower oxygen content $(7-\delta=6.48)$, the VG phase transition behaves analogous to the highly anisotropic $\mathrm{Bi}_{2} \mathrm{Sr}_{2} \mathrm{CaCu}_{2} \mathrm{O}_{8}$ samples, showing a quasi-2D VG transition. For further deoxygenated samples $(7-\delta=6.4)$, the result is consistent with a pure $2 \mathrm{D}$ vortex-glass model similar to that observed in the even more anisotropic $\mathrm{Tl}_{2} \mathrm{Ba}_{2} \mathrm{CaCl}_{2} \mathrm{O}_{8}$ thin films. The estimated value of the anisotropy in high-quality oxygen-depleted samples is comparable to that of the highly anisotropic superconductors. [S0163-1829(99)05246-7]
\end{abstract}

\section{INTRODUCTION}

The dynamic behavior of the vortex system in hightemperature superconductors (HTS's) is strongly affected by the combined effect of pinning, thermal fluctuations, anisotropy, and dimensionality. ${ }^{1}$ Thermal fluctuations are responsible for the existence of a first-order vortex lattice melting transition $^{2,3}$ in clean systems (untwinned single crystals ${ }^{4-6}$ ) separating two distinct phases, the vortex solid and the vortex liquid. Although the accurate mechanism by which the vortex lattice melts is still unknown, the semiquantitative approach based on the Lindemann criterion assumes that melting occurs when the mean-squared amplitude of fluctuations $\left\langle u^{2}\left(T_{m}\right)\right\rangle^{1 / 2}$ is some fraction $C_{L}$ (the Lindemann constant) of the intervortex distance $a_{0} \cdot{ }^{1}$ It has been proposed that, in the presence of point disorder, the long-range order of the Abrikosov lattice is destroyed and the vortex solid becomes a glass. The glass transition becomes a true secondorder transition ${ }^{7-9}$ such that, in its neighborhood, physical quantities are known to scale with the vortex-glass coherence length and the characteristic relaxation time. Scaling laws have been proposed to collapse $\rho$ - $j$ curves onto a single master curve for temperatures within the critical region in terms of critical exponents $\mathrm{v}, z$, and $D$. The static exponent $\mathrm{v}$ accounts for the divergence of the vortex correlation length, and the dynamic exponent $z$, or more precisely the product
$\mathrm{V} z$, accounts for the divergence of the relaxation time. $D$ is the dimensionality of the transition. Correlated disorder, on the other hand, stabilizes a distinct low-temperature vortex phase, the Bose glass, and a different interpretation of the second-order transition into the liquid state has been proposed by Nelson and Vinokur in the frame of Bose-glass theory. ${ }^{10,11}$ Very recently, experimental evidence has been presented for the existence of a Bose-glass phase in twinned YBCO single crystals. ${ }^{12}$ This suggests that twin boundaries, often present in thin films, might stabilize a Bose glass instead of a three-dimensional (3D) vortex glass. For magnetic fields parallel to the $c$ axis, Bose-glass theory predicts critical scaling laws similar to those of the 3D vortex glass. Exponents $z^{\prime}$ and $v^{\prime}$ are defined which describe the divergence of the transverse wandering of a localized vortex line $\left(l_{\perp}\right), l_{\perp}$ $=1 /\left(T_{\mathrm{BG}}-T\right)^{\mathrm{v}^{\prime}}$, and of the relaxation time of a fluctuation with this size $\tau \approx l_{\perp}^{z^{\prime}}$, as the transition temperature $T_{\mathrm{BG}}$ is approached.

An interesting feature of the vortex matter is the change of dimensionality of the vortex-glass $(\mathrm{VG})$ transition when the magnetic field is increased. A dimensional crossover from a 3D to a quasi-2D vortex system with a finite glass transition temperature has been predicted ${ }^{7,8,13,14}$ to occur above a critical magnetic field. At this field the vortex correlation length becomes comparable to cell dimensions. This 
transition, controlled by the anisotropy, occurs at unattainably high fields in YBCO, but it has been observed in the more anisotropic BSCCO. ${ }^{15}$ It has been proposed that $3 \mathrm{D}$ $(D=3)$ and quasi-2D $(D=2)$ transitions obey the same scaling laws, but with different values for the parameter $D$. For even higher values of the anisotropy, like those of $\mathrm{Tl}_{2} \mathrm{Ba}_{2} \mathrm{CaCu}_{2} \mathrm{O}_{8}$, a pure 2D glass transition has been reported to occur at high fields. ${ }^{16}$ While in $3 \mathrm{D}$ or quasi-2D the vortexglass transition has been proposed to occur at finite temperature $T_{g}$, a pure $2 \mathrm{D}$ transition should take place at a zero glass transition temperature $T_{g}=0 .^{7,8,17}$ In this case vortex lengths are smaller than the separation between nonconsecutive planes. It is clear, then, that anisotropy plays a central role in determining the dimensionality of glass transition. Large values of anisotropy and short coherence lengths act to reduce vortex length and to enhance the size of the critical regime. The dynamics of the vortex system is strongly influenced by the coupling properties of vortices in adjacent planes, ${ }^{14,18}$ and the anisotropy parameter $\gamma$ determines to which extent vortices are correlated.

While for fully oxygenated YBCO, the anisotropy parameter $\gamma$ takes values between 5 and 7 , and the vortices are strongly correlated defining a relatively narrow critical regime, $\gamma$ is known to increase when the oxygen content is reduced. In this frame, recent studies ${ }^{19}$ of the anisotropy parameter $\gamma=\left(\rho_{c} / \rho_{a}\right)^{1 / 2}$ in terms of the resistivity values measured close to $T_{c}$ along the $c$ axis $\left(\rho_{c}\right)$ and in plane $\left(\rho_{a}\right)$ have shown $\gamma$ attaining values as high as 60 for $\mathrm{YBa}_{2} \mathrm{Cu}_{3} \mathrm{O}_{6.68}$ single crystals. This value is comparable to those of the highly anisotropic superconductors $\mathrm{Tl}_{2} \mathrm{Ba}_{2} \mathrm{CaCu}_{2} \mathrm{O}_{8}(\gamma \approx 70-150)$ and $\mathrm{Bi}_{2} \mathrm{Sr}_{2} \mathrm{CaCu}_{2} \mathrm{O}_{8}(\gamma \approx 50-$ 130). However, the corresponding scaling behavior and the dimensionality of the glass transition have not been analyzed so far up to the lowest oxygen contents. Hou et al. ${ }^{20}$ have reported 3D scaling behavior for oxygen-depleted samples up to $T_{c}$ values of $48.5 \mathrm{~K}$, inferring smaller values $(\gamma \approx 30$ ) of the anisotropy. Although the vortex system obeyed a $3 \mathrm{D}$ scaling analysis with reasonable values of the scaling parameters for these moderately oxygen-depleted films, a deviation of a VG boundary $H_{g}(T)$ from a $\left(1-T / T_{c}\right)^{3 / 2}$ temperature dependence was also observed, ${ }^{20}$ suggesting that the flux dynamics might change from three dimensional to quasi two dimensional at high fields. Similar observations have been reported on the irreversibility line in $\mathrm{Sm}_{1.85} \mathrm{Ce}_{0.15} \mathrm{CuO}_{4-x}$ and Pr-doped YBCO single crystals. ${ }^{21,22}$

In this paper, we present results of the vortex-glass-liquid transition on high-quality epitaxial $\mathrm{YBa}_{2} \mathrm{Cu}_{3} \mathrm{O}_{7-\delta}$ thin films in magnetic fields up to $7 \mathrm{~T}$ applied parallel to the $c$ axis. Scaling analysis of the nonlinear resistivity-current $(\rho-j)$ data demonstrates that, when reducing the oxygen content, the vortex-glass system crosses over from from 3D or Bose glass (for oxygen contents $7-\delta=7-6.75$ ) through quasi-2D $(7-\delta=6.48)$ into a pure $2 \mathrm{D}(7-\delta=6.4)$ vortex glass system with $T g=0 \mathrm{~K}$. A similar behavior has been observed in highly anisotropic $\mathrm{Bi}_{2} \mathrm{Sr}_{2} \mathrm{CaCu}_{2} \mathrm{O}_{8}$ and $\mathrm{Tl}_{2} \mathrm{Ba}_{2} \mathrm{CaCu}_{2} \mathrm{O}_{8}$ thin films, ${ }^{15,16}$ and interestingly, our analysis shows that oxygendepleted samples have anisotropy values not far from those of the strongly anisotropic superconductors.

\section{EXPERIMENT}

The samples for this study were high-quality epitaxial $\mathrm{YBa}_{2} \mathrm{Cu}_{3} \mathrm{O}_{7-\delta}$ films grown $c$ oriented on (100) $\mathrm{SrTiO}_{3}$ using

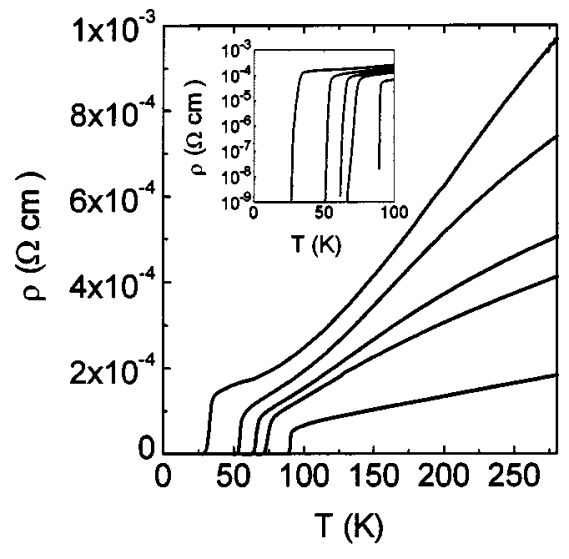

FIG. 1. Electrical resistivity vs temperature for high-quality epitaxial $\mathrm{YBa}_{2} \mathrm{Cu}_{3} \mathrm{O}_{7-\delta}$ thin films at zero magnetic field with values of $7-\delta$ ranging from 7 to 6.4 .

a high-pressure (3.6 mbar pure oxygen) sputtering system. The substrate temperature was $800^{\circ} \mathrm{C}$, and the oxygen content was adjusted in situ following a stability line of the pressure-temperature phase diagram ${ }^{23}$ during sample cool down $(1 \% \mathrm{~min})$. The film thickness was kept in the range 500-700 $\AA$ to ensure a homogeneous oxygen distribution. $I-V$ curves were measured on photolithographically patterned bridges with dimensions $30 \times 400 \mu \mathrm{m}^{2}$. Contacts were done on evaporated silver pads which ensured small contact resistances. A temperature stability better than $50 \mathrm{mK}$ was attained prior to data acquisition. A magnetic field in the range 0-7 $\mathrm{T}$ was applied perpendicular to the sample (parallel to the $c$ axis).

\section{RESULTS AND DISCUSSION}

Figure 1 shows resistivity vs temperature curves at zero field for various oxygen contents. The inset displays the resistive transitions in a logarithmic scale to demonstrate the high quality of our samples. Besides narrow transitions, small values of the residual resistance can be seen, smaller by more than a factor of 2 than previously reported data for thin films with a similar oxygen content. ${ }^{23}$ Interestingly, our residual resistivity values compare well with the values reported for high-quality single crystals, ${ }^{19}$ which points to a very homogeneous oxygen distribution and a very ordered oxygen structure.

$I-V$ curves were first measured in fully oxygenated and slightly oxygen-depleted samples. The vortex-glass phase transition was analyzed using the scaling relation

$$
E(J) \sim J \xi_{g}^{D-2-z} E_{ \pm}\left[J \xi_{g}^{D-1} \phi_{0} / K_{B} T\right],
$$

where $\tau_{g} \sim\left|T-T_{g}\right|^{-z \vee}$ is the vortex-glass coherence length, $\mathrm{V}$ and $z$ are the static and dynamic exponents, respectively, the parameter $D$ is the dimension of the system, and $E_{ \pm}$is a universal scaling function above $\left(E_{+}\right)$and below $\left(E_{-}\right) T_{g}$. In every case the scaling exponents took values well in the range reported previously for the corresponding oxygen contents and the dimension parameter $D$ was always 3 . Typical results for oxygen contents $7-\delta=7$ and $7-\delta=6.75$ are collected in Table I. Scaling exponents were independent of the magnetic field up to $7 \mathrm{~T}$ applied parallel to the $c$ axis. Since YBCO in-plane lattice parameters are very close, domains 
TABLE I. Critical scaling exponents $v$ and $z$ for $\mathrm{YBa}_{2} \mathrm{Cu}_{3} \mathrm{O}_{7-\delta}$ films with various oxygen contents, in different magnetic fields obtained from $\rho(j)$ curves, including the VG transition $T_{g}$ and the values of dimensionality $D$.

\begin{tabular}{cccccc}
\hline \hline $7-\delta$ & $H(\mathrm{~T})$ & $D$ & $T_{g}(\mathrm{~K})$ & $\mathrm{v}$ & $z$ \\
\hline 7 & 2 & 3 & 86.50 & 1.4 & 4.85 \\
& 2 & Bose glass & 86.5 & 1.0 & 7.4 \\
& 6 & 3 & 75.33 & 1.4 & 4.87 \\
& 6 & Bose glass & 75.33 & 1.0 & 7.5 \\
6.75 & 1 & 3 & 65.43 & 1.8 & 3.9 \\
& 1 & Bose glass & 65.43 & 1.0 & 6.35 \\
& 5 & 3 & 51.20 & 1.83 & 4.0 \\
& 5 & Bose glass & 51.20 & 1.0 & 6.5 \\
6.48 & 5 & 2 & 13.73 & 1.89 & 3.95 \\
& 6 & 2 & 10.46 & 1.9 & 3.85 \\
6.4 & 0.5 & 2 & 17.42 & 1.83 & 3.87 \\
& 1 & 2 & 13.57 & 1.83 & 3.84 \\
& 2 & & Pure 2D VG transition, $T_{g}=0$ & \\
& 7 & \multicolumn{5}{c}{ Pure 2D VG transition, $T_{g}=0$} & \\
\hline \hline
\end{tabular}

rotated $90^{\circ}$ frequently appear in thin epitaxial films grown on $\mathrm{SrTiO}_{3}$, giving rise to dense arrays of twin boundaries. Twin boundaries may act as correlated disorder stabilizing a Boseglass phase as quoted in the Introduction. Bose-glass theory ${ }^{10,11}$ predicts that, within the critical region, the current density and electric field obey the following scaling relation:

$$
E\left|\frac{T-T_{\mathrm{BG}}}{T_{\mathrm{BG}}}\right|^{-\mathrm{v}^{\prime}\left(z^{\prime}-1\right)}=F_{ \pm}\left(\left|\frac{T-T_{\mathrm{BG}}}{T_{\mathrm{BG}}}\right|^{-3 \mathrm{v}^{\prime}} J \Phi_{0} / c\right),
$$

where $F_{ \pm}$are universal scaling functions above and below the transition temperature. Accordingly, plots of

$$
\frac{V}{I}\left|\frac{T-T_{\mathrm{BG}}}{T_{\mathrm{BG}}}\right|^{-\mathrm{v}^{\prime}\left(z^{\prime}-2\right)} \text { versus } I\left|\frac{T-T_{\mathrm{BG}}}{T_{\mathrm{BG}}}\right|^{-3 \mathrm{v}^{\prime}},
$$

collapse $I-V$ data in two master curves. Critical exponents $z^{\prime}=7-8$ and $v^{\prime}=1$ were obtained for the fully oxidized sample and for $7-\delta=6.75$, which produced a very good collapse of the $I-V$ curves (see Table I). These values are well in the range reported previously by Nelson and Vinokur ${ }^{11}$ from the scaling analysis of thin film samples. It is, therefore, not possible to distinguish between a $3 \mathrm{D}$ vortex-glass and a Bose-glass transition in terms of the scaling analysis.

Samples with smaller oxygen content were analyzed to investigate the existence of a quasi-2D regime. Figure 2(a) shows the typical nonlinear $I-V$ characteristics on a doublelogarithmic scale for a $\mathrm{YBa}_{2} \mathrm{Cu}_{3} \mathrm{O}_{6.48}\left(T_{c}=47 \mathrm{~K}\right)$ sample in a magnetic field of $5 \mathrm{~T}$ applied parallel to the $c$ axis. The range of these $I-V$ isotherms is from $5 \mathrm{~K}$ at the lower right to $25 \mathrm{~K}$ at the upper left in increments of $1 \mathrm{~K}$, respectively. While clear positive curvatures are observed at intermediate temperatures, the negative curvature in the high-current regime at low temperatures can be ascribed to the glass phase for which resistivity displays a dependence on current in the form $\exp \left[-\left(j_{0} / j\right)^{\mu}\right]$ with a positive value of the exponent $\mu$. It is well known that approaching $T_{g}$ the exponent $\mu$ charac-
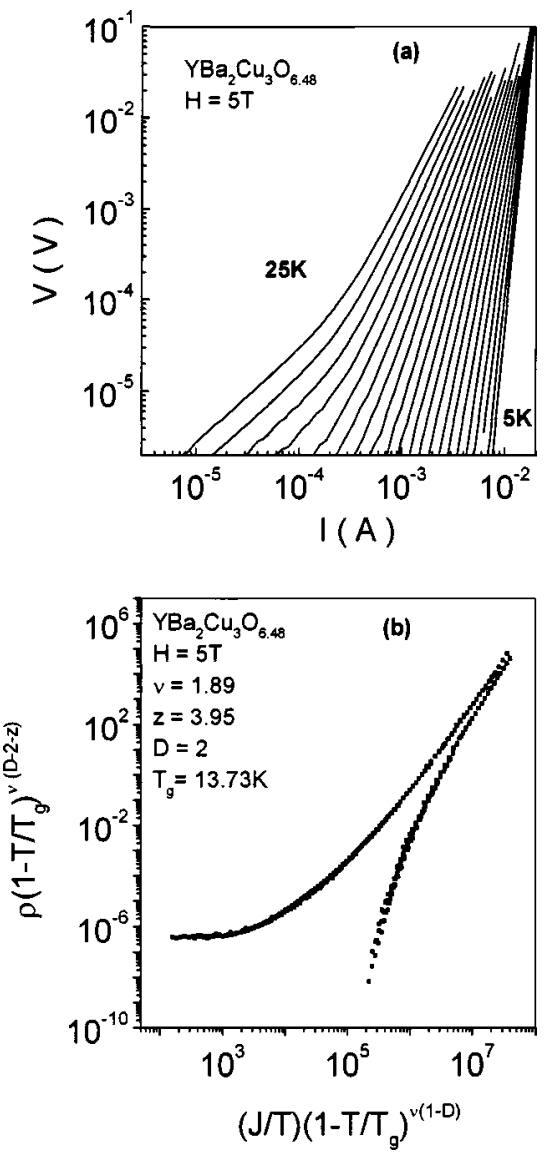

FIG. 2. (a) $I-V$ characteristics in double-logarithmic scale for $\mathrm{YBa}_{2} \mathrm{Cu}_{3} \mathrm{O}_{6.48}$ in a magnetic field of $5 \mathrm{~T}$. The temperature ranges are from $5 \mathrm{~K}$ (lower right) to $25 \mathrm{~K}$ (upper left) in increments of $1 \mathrm{~K}$. (b) $\rho^{*}-j^{*}$ scaling curves according to a quasi-2D VG model for $\mathrm{YBa}_{2} \mathrm{Cu}_{3} \mathrm{O}_{6.48}$ in a magnetic field of $5 \mathrm{~T}$.

terizing the vortex-glass phase becomes vanishingly small and the slope of the critical isotherm fulfills the following relation: ${ }^{7,8}$

$$
\rho\left(j, T_{g}\right) \sim j^{\alpha},
$$

providing a possibility of obtaining $z$ (independent of scaling), since $\alpha=(z+2-D) /(D-1)$, where $D$ is the dimensionality of the system and $z$ is predicted to be in the range of 4-6. An independent measurement of $T_{g}$, on the other hand, is obtained from the zero extrapolation of $(d \ln \rho / d T)^{-1}$ plots. According to this, we find $T_{g}=14 \pm 0.3 \mathrm{~K}$ and $\alpha=3.95$. If we interpret the result as a $3 \mathrm{D}$ vortex-glass transition, i.e., $D$ $=3$, we obtain an unreasonably high value of $z=8.9$. However, assuming a quasi-two-dimensional vortex-glass transition with $D=2, z=3.95$ is obtained, well in the range of variation for $\alpha$. Using $D=2$ and $z=3.95 \pm 0.2$, a good scaling was obtained with $\mathrm{V}=1.89 \pm 0.1$ over a critical regime more than $20 \mathrm{~K}$ wide. The critical region is wider than the one observed in a $3 \mathrm{D}$ vortex system $(5 \mathrm{~K})$, due to an enhanced effect of fluctuations caused by the weakened interlayer coupling. ${ }^{1}$ Attempts to scale $I-V$ curves according to a Bose-glass transition produced also unrealistic values of the exponents $\left(z^{\prime}=13.05, \mathrm{v}^{\prime}=0.68\right.$, and $\left.T_{\mathrm{BG}}=13.41 \mathrm{~K}\right)$, which allows discarding this mechanism for the vortex-solid-liquid transition. Figure 2(b) shows how the $\rho(j)$ data collapse into 

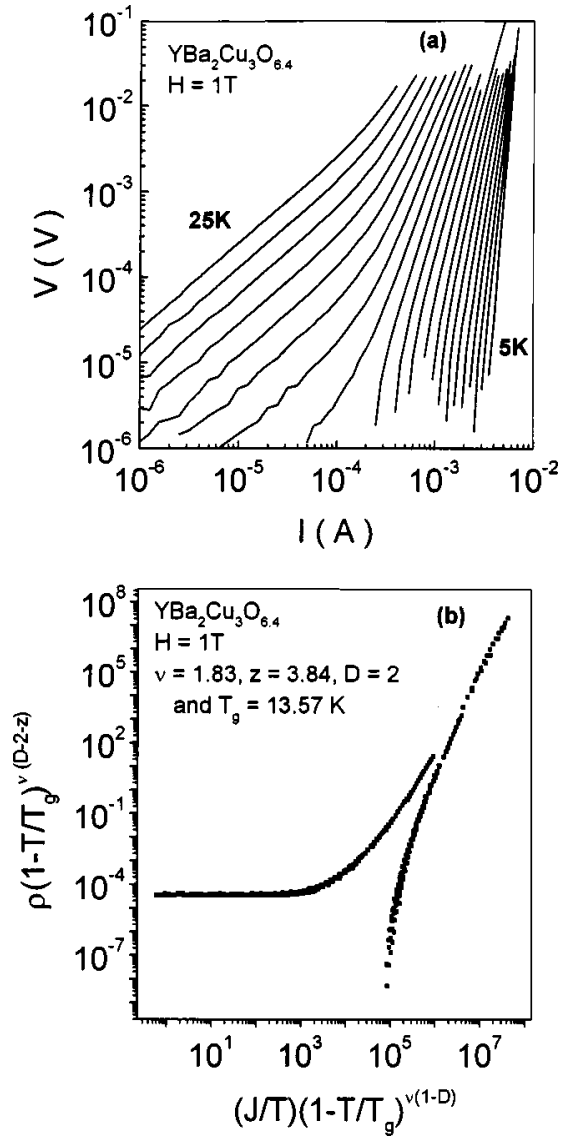

FIG. 3. $\rho^{*}-j^{*}$ scaling curves according to a quasi-2D VG model for $\mathrm{YBa}_{2} \mathrm{Cu}_{3} \mathrm{O}_{6.4}$ in a magnetic field of $1 \mathrm{~T}$.

two universal scaling functions by plotting the $\rho(j)$ data as $\rho^{*}=\rho\left(1-T / T_{g}\right)^{-\mathrm{v} z}$ versus $J^{*}=(J / T)\left(1-T / T_{g}\right)^{-\mathrm{v}}$. It is remarkable that the critical exponents coincide with those obtained for our samples with small oxygen deficiencies ( 7 $-\delta=6.75)$ from 3D scaling analysis and are also in good agreement with the values reported previously. ${ }^{9}$ We found the same exponents for magnetic fields in the range 4-6 T, again pointing to the independence of the critical exponents on magnetic field as reported recently. ${ }^{24}$ For smaller fields up to $1 \mathrm{~T}$, however, scalings with $D=2$ and same exponents were somewhat poorer, but still rather high values of $z$ $=7.7$ were obtained with $D=3$. This might be indicative that the crossover field to the quasi-2D glass transition might be below $1 \mathrm{~T}$ for this oxygen doping level.

Since we found a quasi-2D scaling for samples with an oxygen content of $7-\delta=6.48$ at high fields, we further reduced the oxygen content to investigate the dependence of the scaling parameters and, eventually, of the transition dimensionality on the oxygen content. Shown in Fig. 3(a) are the $I-V$ isotherms for a $\mathrm{YBa}_{2} \mathrm{Cu}_{3} \mathrm{O}_{6.4}$ film measured in a field of $1 \mathrm{~T}$. A well-behaved quasi-2D scaling [Fig. 3(b)] with values of the critical exponents similar to those found for $\mathrm{YBa}_{2} \mathrm{Cu}_{3} \mathrm{O}_{6.48}$ at high fields $(D=2, \mathrm{v}=1.8 \pm 0.2$, and $z$ $=3.8 \pm 0.2$ ) was observed in the small magnetic fields of 0.5 and $1 \mathrm{~T}$. We again found that both static $\mathrm{V}$ and dynamic $z$ exponents are independent of magnetic field and oxygen content. While it is already known that the critical exponents are field independent for a 3D vortex-glass transition ${ }^{24}$ (in agreement with our results in Table I for $\mathrm{YBa}_{2} \mathrm{Cu}_{3} \mathrm{O}_{7}$ and
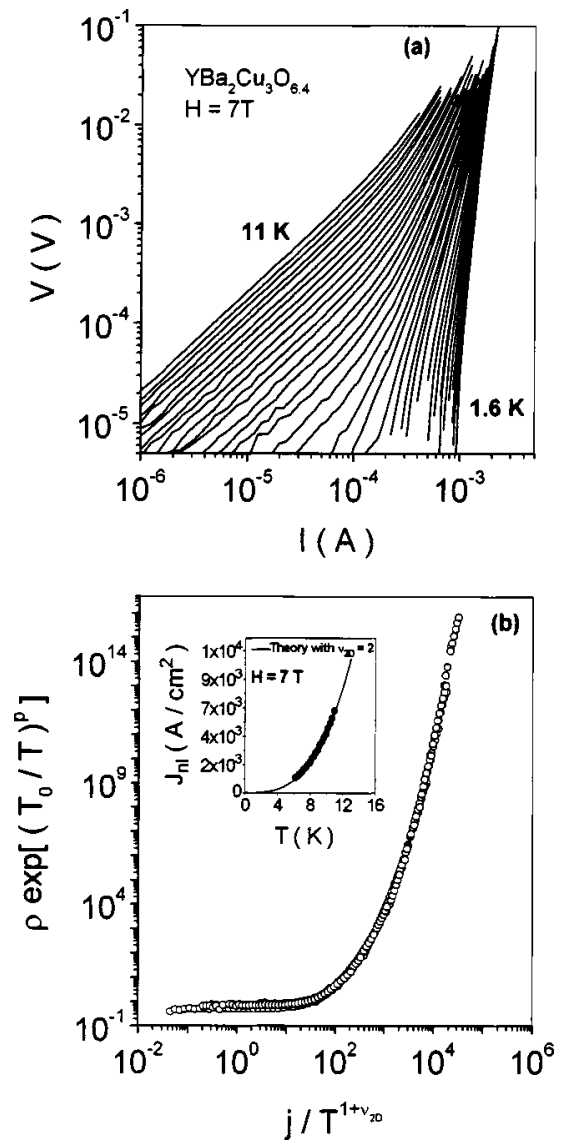

FIG. 4. (a) $I-V$ characteristics in double-logarithmic scale for $\mathrm{YBa}_{2} \mathrm{Cu}_{3} \mathrm{O}_{6.4}$ in a magnetic field of $7 \mathrm{~T}$. The temperature ranges are from $1.6 \mathrm{~K}$ (lower right) to $11 \mathrm{~K}$ (upper left) in increments of $0.2 \mathrm{~K}$. (b) $\rho$ - $j$ scaling curves according to a pure $2 \mathrm{D}$ VG theory, for $H$ $=7 \mathrm{~T}$, with parameters $\mathrm{V}_{2 \mathrm{D}}=2, p=0.78$, and $T_{0}=230$. Inset: temperature dependence of the nonlinear current density $j_{\mathrm{nl}}(T)$ for $H=7 \mathrm{~T}$ (solid circles). The solid line represents the theoretical fit according to the $2 \mathrm{D} V \mathrm{VG}$ power law with $\mathrm{V}_{2 \mathrm{D}}=2$.

$\mathrm{YBa}_{2} \mathrm{Cu}_{3} \mathrm{O}_{6.75}$ ), our additional observation for the quasi-2D system extends the concept of universality of the scaling parameters proposed by Moloni et al. ${ }^{24}$ for 3D deoxygenated samples in terms only of the magnetic field.

When magnetic field is increased above $1 \mathrm{~T}$ in those samples with oxygen content $7-\delta=6.4$, the vortex system shows a crossover into a pure $2 \mathrm{D}$ vortex-glass transition. Figures 4(a) and 5(a) display the nonlinear $I-V$ characteristics on a double-logarithmic scale for a $\mathrm{YBa}_{2} \mathrm{Cu}_{3} \mathrm{O}_{6.4}$ thin film measured in fields of 7 and $2 \mathrm{~T}$, respectively. The temperature ranges between 1.6 and $11 \mathrm{~K}$ in $0.2-\mathrm{K}$ steps in Fig. 4(a) and between $3 \mathrm{~K}$ at the lower right and $20 \mathrm{~K}$ at the upper left in increments of $0.5 \mathrm{~K}$ in Fig. 5(a). For magnetic fields higher than $2 T$ the quasi-2D scaling analysis of the $\mathrm{YBa}_{2} \mathrm{Cu}_{3} \mathrm{O}_{6.4}$ sample yields unphysical high values of $z$, suggesting that the VG transition may cross over into a pure 2D character. In the case of a pure two-dimensional, i.e., completely decoupled, vortice along the $c$-axis direction, the system is known never to be in a glassy state unless $T=0$, i.e., $T_{g}=0 \mathrm{~K}$. Direct evidence for this behavior was obtained from $\rho \exp \left[\left(T_{0} / T\right)^{p}\right]$ versus $j / T_{2 \mathrm{D}}^{1+\mathrm{v}}$ scaling plots, as proposed by Dekker $e t a l .{ }^{25}$ In these expressions $\rho$ is the resistivity, $j$ is the measuring current density, $T_{0}$ is related to the vortex line 

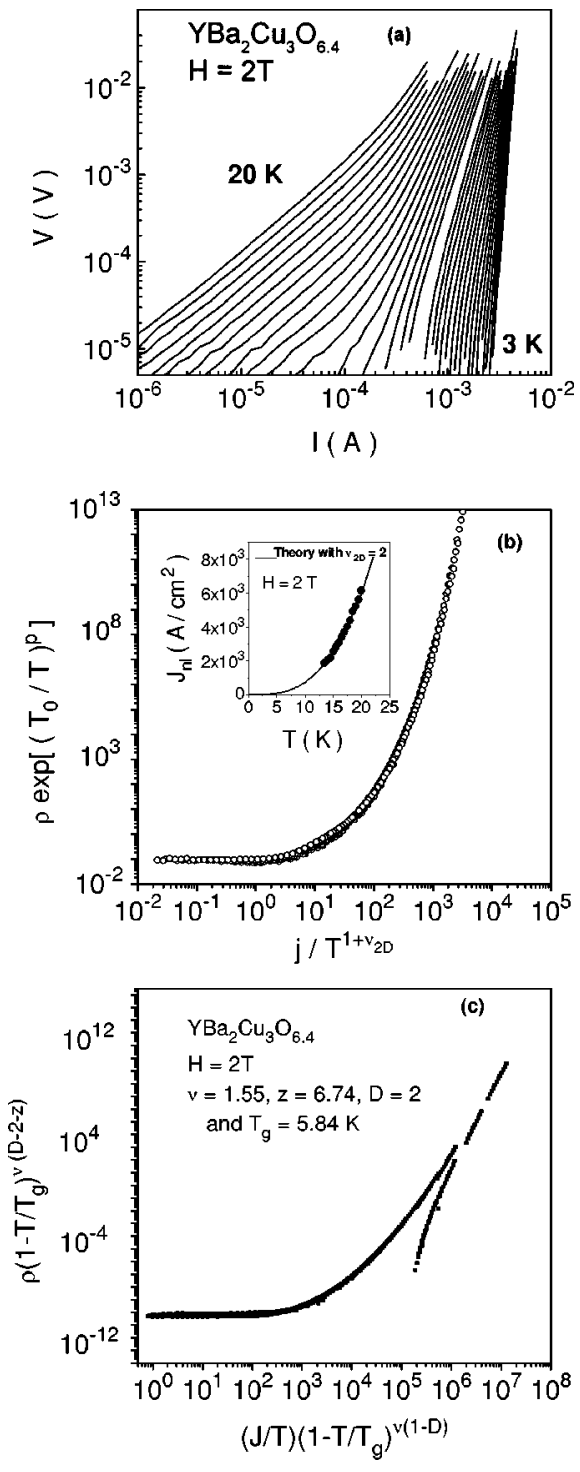

FIG. 5. (a) $I-V$ characteristics in double-logarithmic scale for $\mathrm{YBa}_{2} \mathrm{Cu}_{3} \mathrm{O}_{6.4}$ in a magnetic field of $2 \mathrm{~T}$. The temperature ranges are from $3 \mathrm{~K}$ (lower right) to $20 \mathrm{~K}$ (upper left) in increments of $1 \mathrm{~K}$. (b) $\rho$ - $j$ scaling curves according to a pure 2D VG theory, for $H$ $=2 \mathrm{~T}$, with parameters $\mathrm{V}_{2 \mathrm{D}}=2, p=1$, and $T_{0}=230$. Inset: temperature dependence of the nonlinear current density $j_{\mathrm{nl}}(T)$ for $H$ $=2 \mathrm{~T}$ (solid circles). The solid line represents the theoretical fit according to the $2 \mathrm{D} V \mathrm{VG}$ power law with $\mathrm{V}_{2 \mathrm{D}}=2$. (c) $\rho$ - $j$ scaling curves according to a quasi-2D VG theory, for $H=2 \mathrm{~T}$, with parameters $\mathrm{v}=1.55, z=6.74, D=2$, and $T_{g}=5.84 \mathrm{~K}$.

energy, and $p$ and $\mathrm{v}_{2 \mathrm{D}}$ are the characteristic exponents of the 2D transition. The excellent scaling shown in Fig. 4(b) for 7 T suggests a pure 2D vortex-glass phase transition with $T_{g}$ $=0 \mathrm{~K}$, with parameters $\mathrm{V}_{2 \mathrm{D}}=2, T_{0}=230 \mathrm{~K}$, and $p=0.78$. A good $2 \mathrm{D}$ scaling with parameters $\mathrm{v}_{2 \mathrm{D}}=2, T_{0}=230 \mathrm{~K}$, and $p=1$ was also obtained for the 2-T field [see Fig. 5(b)]. According to the theory of the $2 \mathrm{D}$ VG transition, the vortexglass correlation length diverges as

$$
\xi_{2 \mathrm{D}}=a_{0}\left(\varepsilon_{0} d / k_{B} T\right)^{\mathrm{V} 2 \mathrm{D}}
$$

where $\mathrm{v}_{2 \mathrm{D}}$ is the $2 \mathrm{D}$ VG exponent, $a_{0}$ the intervortex spacing, and $\varepsilon_{0} d$ the core energy of vortex segment of length $d$. In presence of an external current density, a crossover from linear to nonlinear behavior is expected to occur at $j=j_{\mathrm{nl}}$, when the energy $j_{\mathrm{nl}} \phi_{0} \xi_{2 \mathrm{D}}$ necessary to create vortex excitations becomes comparable to the thermal energy $k_{B} T$. Combining this dependence with Eq. (3), one finds

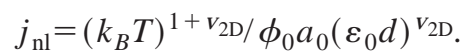

The insets of Figs. 4(b) and 5(b) show the $j_{\mathrm{nl}}$ data defined as the value of the current density at which the resistivity deviates by $5 \%$ from its constant linear value together with a fit to Eq. (4), which yielded an exponent $\mathrm{v}_{2 \mathrm{D}}=2$, in agreement with a pure $2 \mathrm{D} V \mathrm{VG}$ model. Since the quasi-2D regime is unambiguously established for fields equal or lower than $1 \mathrm{~T}$ and the pure 2D glass transition for fields higher than $2 \mathrm{~T}$, it may be interesting to investigate whether the quasi-2D scaling analysis accounts for the crossover to the pure 2D behavior. Although quasi-2D scaling yielded unphysical values for the critical exponent $z$ in the high-field region, at a magnetic field of $2 \mathrm{~T}$, close to the crossover, a reasonable quasi-2D scaling is obtained with low $T_{g}\left(T_{g}=5 \mathrm{~K}\right)$ and a dynamic exponent $z$ of 6.74 [see Fig. 5(c)]. This value, although large, is not far from the range $4-6$ predicted by the theory. ${ }^{8}$ This seems to support the point that the quasi-2D vortex-glass transition merges into the pure 2D when the glass transition temperature approaches zero. The crossover into a regime with reduced dimensionality might then reflect in an increase of the dynamic exponent $z$.

So far, we have shown that the VG transition changes from 3D (or Bose glass) to 2D when the oxygen content is reduced. An estimate of the vortex length for the different cases can be done to show that the dimensionality of the transition is linked to the degree of coupling between $\mathrm{CuO}$ planes reflected in the vortex length. It seems reasonable that the strong anisotropy of these materials affects the vortexglass correlation length $\xi_{g}$, and its divergence near $T_{g}$ results from the divergence of the individual correlation lengths parallel and perpendicular to the $c$ axis $\left(\xi_{c}\right.$ and $\xi_{a b}$, respectively). Following the reasoning by Yamasaki et al., ${ }^{15}$ in the case of a quasi-2D VG transition, a reduced vortex length precludes $\xi_{c}$ to diverge (and remains practically constant). The divergence of $\xi_{g}$ is then restricted to $\xi_{a b}$. The current density $j_{x}^{+}$at the onset of the nonlinear behavior can be used to extract an upper limit for the vortex correlation length along the $c$ axis. Since the nonlinear behavior shows up when the work done by the Magnus force to create vortex excitation equals the thermal energy, the following relation holds: $j_{x}^{+} \phi_{0} \xi_{c} \xi_{a b} \approx k_{B} T .^{7-9}$ For $\mathrm{YBa}_{2} \mathrm{Cu}_{3} \mathrm{O}_{6.4}$ at $1 \mathrm{~T}$ and $T / T_{c}=0.95$, where the critical scaling persists, we obtain $j_{x}^{+} \approx 2.23 \times 10^{4} \mathrm{~A} / \mathrm{cm}^{2}$ and $\xi_{c} \xi_{a b} \approx 740 \mathrm{~nm}^{2}$. Since $\xi_{a b}$ should be definitely larger than the intervortex spacing $\left(a_{0}\right.$ $=455 \AA$ ), assuming $\xi_{a b} \approx 2 a_{0}$, an estimate for $\xi_{c}$ is obtained, $\xi_{c}<80 \AA$, smaller than the film thickness. The same reasoning for the $\mathrm{YBa}_{2} \mathrm{Cu}_{3} \mathrm{O}_{6.48}$ sample at $5 \mathrm{~T}$, where the quasi-2D behavior is also observed, yields $j_{x}^{+} \approx 1.78$ $\times 10^{4} \mathrm{~A} / \mathrm{cm}^{2}$, yielding a vortex length along the $c$ axis, $\xi_{c}$ $<234 \AA$, which is still smaller than sample thickness. A rough estimate of the vortex correlation length along $c$ can also be obtained for the fully oxygenated sample following 
the same reasoning: At a field of $5 \mathrm{~T}$ and for $T / T_{c}=0.94$, $j_{x}^{+} \approx 6.6 \times 10^{3} \mathrm{~A} / \mathrm{cm}^{2}$, and $\xi_{c} \approx 2300 \AA$, considerably larger than sample thickness. It turns out that, in this case, the vortex length along the $c$ axis is limited by sample thickness. It is worth remarking that in the $3 \mathrm{D}$ sample, unlike in the quasi-2D case, $\xi_{c}$ is expected to be strongly temperature dependent. Our estimate has to be considered, then, as a very crude one. However, it is not that far from the vortex length obtained by other authors for a single-crystal base on flux transformer measurements. ${ }^{26}$ The important point is that, while in the 3D sample the vortex correlation length is limited by sample dimensions, in the quasi-2D sample the large anisotropy is responsible for considerably shorter lengths.

We can also obtain an order-of-magnitude estimate of the vortex length in the pure $2 \mathrm{D}$ sample $\mathrm{YBa}_{2} \mathrm{Cu}_{3} \mathrm{O}_{6.4}$ at fields larger than $2 \mathrm{~T}$. The parameter $T_{0}$, obtained from the pure 2D scaling, is interpreted in terms of the vortex core energy. Assuming for the density and mass of the Cooper pairs, $\rho_{s}$ $=10^{27} \mathrm{~m}^{-3}$ and $m=1.82 \times 10^{-30} \mathrm{~kg}$, respectively, we can write an order-of-magnitude estimate for the vortex core energy, $\varepsilon_{0} d=h^{2} \rho_{s} d / m$, for a vortex segment of length $d$. If we set $\varepsilon_{0} d=230 \mathrm{~K}$, the value obtained experimentally for $T_{0}$, a vortex length of $5.2 \AA$ results, in agreement with the $2 \mathrm{D}$ picture. Interestingly, for this vortex length, consecutive $\mathrm{CuO}_{2}$ planes (separated by $\mathrm{Y}^{3+}$ ions) are coupled, and nonconsecutive planes $8 \AA$ apart would then be decoupled. A picture arises that is comparable with the case of one single unit cell of YBCO by Dekker et al. ${ }^{25}$ and not surprisingly their exponent $p=0.6$ and parameter $T_{0}=230$ at $0.5 \mathrm{~T}$ are very similar to ours.

Assuming an approximate value of $1 \mathrm{~T}$ for the crossover field to the pure 2D behavior in the oxygen-depleted $\mathrm{YBa}_{2} \mathrm{Cu}_{3} \mathrm{O}_{6.4}$ sample, an estimate of the anisotropy can be extracted assuming an expression for this critical field $H_{\text {cr }}$ $=\phi_{0} /(\gamma s)^{2}$, where $s$ is the distance between consecutive layer blocks, $\approx 8 \AA$. An anisotropy parameter of 57 is obtained, in good agreement with the values reported by Takenaka et al. ${ }^{19}$ from perpendicular resistivity measurements, and considerably higher than the value predicted from scaling analysis for lower oxygen contents. ${ }^{20}$ Using a more detailed analysis, ${ }^{27}$ integrating over the Fourier spectrum of fluctuations, another estimate of the anisotropy can be obtained $^{28}$ from expression $H_{\mathrm{cr}}=\left(10^{3}-10^{4}\right) / \gamma^{2}$, which yields values of the anisotropy in the range $30-100$. It is worthwhile remarking again that this large anisotropy is not far from those reported for the highly anisotropic superconductors (BSCCO and TBCCO). An interesting possibility, suggested earlier, ${ }^{20}$ is that the anisotropy may be seriously influenced by the oxygen order in the sense that a disordered structure might result in lower anisotropy values.

We want to make the last important remark that, although the 2D glass transition was first reported for one-unit-cell- thick YBCO samples and departures from the 3D scaling were observed for 100 - $\AA$-thick films, ${ }^{25}$ we do not expect the reported behavior to be due to the small thickness of the films. First, we did not observe differences in $\mathrm{YBa}_{2} \mathrm{Cu}_{3} \mathrm{O}_{6.4}$ films with thicknesses changing between 500 and $700 \AA$, necessary to ensure the very homogeneous oxygen content. And, second, experiments conducted on 500-A-thick fully oxygenated and slightly deoxygenated samples did show a well-behaved 3D scaling with values of the critical exponents similar to those found in thicker $(1000 \AA)$ samples $(D=3, \mathrm{v}=1.4$, and $z=4.87)$. The crossover into $2 \mathrm{D}$ behavior found in our deoxygenated samples is then a manifestation of an intrinsic property of the vortex system, and by no means is related to the thickness of the film.

In summary, we have studied the vortex-glass transition in high-quality epitaxial $\mathrm{YBa}_{2} \mathrm{Cu}_{3} \mathrm{O}_{7-\delta}$ thin films in magnetic fields up to $7 \mathrm{~T}$. Our experimental data show clear evidence of a crossover from 3D (or Bose glass) to a pure 2D VG transition with $T_{g}=0 \mathrm{~K}$ when the oxygen content is reduced. For fully oxygenated and slightly oxygen-depleted samples, the scaling analysis of vortex-solid-liquid transition does not allow one to distinguish between a $3 \mathrm{D}$ vortexglass transition or a Bose-glass transition. Although the last possibility is very likely in thin film samples, were twin boundaries are known to form dense arrays, careful measurements as a function of angle (currently out of our possibilities) are necessary to ascertain it. Intermediate oxygen contents $\mathrm{YBa}_{2} \mathrm{Cu}_{3} \mathrm{O}_{6.48}$ show a quasi-2D behavior at high fields. Larger oxygen deficiencies $\left(\mathrm{YBa}_{2} \mathrm{Cu}_{3} \mathrm{O}_{6.4}\right)$ also follow the quasi-2D pattern at low fields $(H<1 \mathrm{~T})$. Scaling parameters $(\mathbf{v}=1.8 \pm 0.2$ and $z=3.8 \pm 0.2)$ in the quasi-2D region were independent of the oxygen content and the magnetic field, extending the concept of universality suggested earlier. ${ }^{24}$ When the field is increased above $1 \mathrm{~T}$, these samples show a crossover to a pure 2D behavior, which follows the scaling laws of the 2D vortex-glass theory. An estimate of the vortex correlation length yields values around $5 \AA$, in agreement with the 2D picture. The anisotropy $(\gamma=30-100)$ deduced for these $\mathrm{YBa}_{2} \mathrm{Cu}_{3} \mathrm{O}_{6.4}$ samples from the crossover field is comparable to the values for the highly anisotropic superconductors and is in agreement with previous measurements of parallel and perpendicular resistivity in deoxygenated YBCO single crystals. ${ }^{19}$

\section{ACKNOWLEDGMENTS}

Z.S. gratefully acknowledges financial support from Agencia Española de Cooperación International (AECI). G.D.L. thanks the sabbaticals SA 106/95 SAB1995-0685 to Universidad Complutense and Ministerio de Educacion y Cultura. Financial support from CICYT Grant No. MAT940604 is also acknowledged. We thank Professor J. L. Vicent for helpful discussions.
*On leave from Universidad del Quindio, Armenia, Colombia.

${ }^{\dagger}$ Author to whom correspondence should be addressed. Electronic address: jacsan@eucmax.sim.ucm.es

${ }^{1}$ G. Blatter, M. V. Feigel'man, V. B. Geshkenbein, A. I. Larkin, and V. M. Vinokur, Rev. Mod. Phys. 66, 1125 (1994).

${ }^{2}$ E. Brezin, D. R. Nelson, and A. Thiaville, Phys. Rev. B 31, 7124 (1985).
${ }^{3}$ D. R. Nelson, Phys. Rev. Lett. 60, 1973 (1988).

${ }^{4}$ H. Safar, P. L. Gammel, D. A. Huse, D. J. Bishop, J. P. Rice, and D. M. Ginsberg, Phys. Rev. Lett. 69, 824 (1992).

${ }^{5}$ M. Charalambous, J. Chaussy, P. Lejay, and V. Vinokur, Phys. Rev. Lett. 71, 436 (1993).

${ }^{6}$ U. Welp, J. A. Fendrich, W. K. Kwok, G. W. Crabtree, and B. W. Veal, Phys. Rev. Lett. 76, 4809 (1996). 
${ }^{7}$ M. P. A. Fisher, Phys. Rev. Lett. 62, 1415 (1989).

${ }^{8}$ D. S. Fisher, M. P. A. Fisher, and D. A. Huse, Phys. Rev. B 43, 130 (1991).

${ }^{9}$ R. H. Koch, V. Foglietti, W. J. Gallagher, G. Coren, A. Gupta, and M. P. A. Fisher, Phys. Rev. Lett. 63, 1511 (1989).

${ }^{10}$ D. R. Nelson and V. M. Vinokur, Phys. Rev. Lett. 68, 2398 (1992).

${ }^{11}$ D. R. Nelson and V. M. Vinokur, Phys. Rev. B 48, 13060 (1993).

${ }^{12}$ S. A. Griguera, E. Morré, E. Osquiguil, C. Balseiro, G. Nieva, and F. de la Cruz, Phys. Rev. Lett. 81, 2348 (1998).

${ }^{13}$ M. V. Feigel'man, V. B. Geshkenbein, and A. I. Larkin, Physica C 167, 177 (1990).

${ }^{14}$ L. I. Glazman and A. E. Koshelev, Phys. Rev. B 43, 2835 (1991).

${ }^{15}$ H. Yamasaki, K. Endo, S. Kosaka, M. Umeda, S. Yoshida, and K. Kajimura, Phys. Rev. B 50, 12959 (1994).

${ }^{16}$ Hai-hu Wen, H. A. Radovan, F.-M. Kamm, P. Ziemann, S. L. Yan, L. Fang, and M. S. Si, Phys. Rev. Lett. 80, 3859 (1998).

${ }^{17}$ M. P. A. Fisher, T. A. Tokuyasu, and A. P. Young, Phys. Rev. Lett. 66, 2931 (1991).

${ }^{18}$ L. L. Daemen et al., Phys. Rev. Lett. 70, 1167 (1993).

${ }^{19}$ K. Takenaka, K. Mizuhashi, T. Takagi, and S. Uchida, Phys. Rev. B 50, 6534 (1994).
${ }^{20}$ Lifang Hou, J. Deak, P. Metcalf, M. McElfresh, and G. Preosti, Phys. Rev. B 55, 11806 (1997).

${ }^{21}$ C. C. Almasan, M. C. de Andrade, Y. Dalichaouch, J. J. Neumeier, C. L. Seaman, M. B. Maple, R. P. Guertin, M. V. Kuric, and J. C. Garland, Phys. Rev. Lett. 69, 3812 (1992).

${ }^{22}$ M. P. Maple, C. C. Almasan, C. L. Seaman, S. H. Han, K. Y. Yoshiara, M. Buchgeister, L. M. Paulius, B. W. Lee, D. A. Gajewski, R. F. Jarrdim, C. R. Fincher, Jr., Garciela B. Blanchet, and R. P. Guertin, J. Supercond. 7, 97 (1994).

${ }^{23}$ E. Osquiguil, M. Maenhoudt, B. Wuyts, and Y. Bruynseraede, Appl. Phys. Lett. 60, 1627 (1992).

${ }^{24}$ Katerina Moloni, Mark Friesen, Shi Li, Victor Souw, P. Metcalf, and M. McElfresh, Phys. Rev. B 56, 14784 (1997).

${ }^{25}$ C. Dekker, W. Eidellooth, and R. H. Koch, Phys. Rev. Lett. 68, 3347 (1992).

${ }^{26}$ D. López, E. F. Righi, G. Nieva, F. de la Cruz, W. K. Kwok, J. A. Fendrich, G. W. Grabtree, and L. Paulius, Phys. Rev. B 53, R8895 (1996).

${ }^{27}$ M. Tinkham, Introduction to Superconductivity, 2nd ed. (McGraw-Hill, New York, 1996).

${ }^{28}$ L. Y. Glazman and A. E. Koshelev, Phys. Rev. B 43, 2835 (1991). 